\title{
sciendo
}

\section{Changes in Lower-Limb Biomechanics, Soft Tissue Vibrations, and Muscle Activation During Unanticipated Bipedal Landings}

\author{
by \\ Shen Zhang', Weijie Fu' ${ }^{1}, \mathrm{Yu} \mathrm{Liu}^{1}$
}

\begin{abstract}
We aimed to explore the biomechanical differences between the anticipated drop jump and unanticipated drop landing. Twelve male collegiate basketball players completed an anticipated drop jump and unanticipated drop landing with double legs from a height of $30 \mathrm{~cm}$. Kinematics, impact force, soft tissue vibrations, and electromyographic (EMG) amplitudes of the dominant leg were collected simultaneously. The anticipated drop jump showed more flexed lower limbs during landing and increased range of motion compared to the unanticipated drop landing. The anticipated drop jump also had lower impact force, lesser soft tissue vibration, and a greater damp coefficient at the thigh muscles compared with the unanticipated drop landing. Significant increases in the EMG amplitudes of the tibialis anterior, lateral gastrocnemius, rectus femoris, and biceps femoris were observed in the anticipated drop jump during the pre/post-activation and downward phases. The anticipated drop jump presented more optimized landing posture control with more joint flexion, lower impact force, less soft tissue vibrations, and full preparation of muscle activations compared with the unanticipated drop landing.
\end{abstract}

Key words: anticipated drop jump, unanticipated drop landing, impact forces, soft tissue vibrations, muscle activation.

\section{Introduction}

During bipedal landings, the peak magnitude of the impact force ranges from three to seven times the body weight (BW) (Yeow et al., 2011a). Numerous studies have reported a close relationship between high impact forces and lower extremity injuries during intensive landings, indicating that the excessive repetitive loading can induce acute injuries such as sprains, muscle-tendon strains or even fractures (Beynnon et al., 2005; Del Coso et al., 2018) and overuse damage such as stress fractures and patellofemoral pain syndrome (Borowski et al., 2008; Dick et al., 2007). To date, the major factors known to affect impact forces have been drop heights / touchdown velocities (Brüggemann et al., 2011; Zhang et al., 2000), landing surfaces / footwear (Dixon et al., 2000; Fu et al., 2013), joint geometry (Shultz et al., 2011), and muscle activation (Santello, 2005; Gołaś et al., 2017).
Among them, studies on the effects of different posture control such as anticipated or unanticipated, on impact forces and corresponding landing biomechanics, are rare, yet important.

Currently, anticipated self-initiated landings, including a landing after stepping off a box (Decker et al., 2003) or unhanding a bar (Self and Paine, 2001), have been widely used to explore the posture control mechanism. A safety or successful landing after a drop, such as a drop jump or a drop landing, requires the body to actively control the segments to attenuate the impact forces when initially contacting the ground and to dissipate the kinetic energy (Moran and Wallace, 2007). This procedure is achieved mostly through changes in lower extremity mechanics (Tamura et al., 2016) such as increased knee flexion to reduce the potential damage to the anterior cruciate ligament (ACL) (Delahunt et al., 2006;

1 - Key Laboratory of Exercise and Health Sciences of the Ministry of Education, Shanghai University of Sport, China. 
Mandelbaum et al., 2005), or an ideal range of leg stiffness to attenuate the shock to avoid bony injuries such as knee osteoarthritis and stress fractures (Butler et al., 2003). Overall, these biomechanical changes in jumping or landing tasks are all controlled by anticipated movement control patterns (Santello, 2005). However, lower extremity injuries such as ankle sprains and ACL tears, may largely occur in certain unexpected circumstances where the human body does not have adequate time to prepare for a landing impact (Almonroeder et al., 2015; Jones and Watt, 1971; Stephenson et al., 2018). Logically, selfinitiated falls and unexpected falls are characterized by different patterns of movement control strategies that may induce various musculoskeletal injuries (Teh et al., 2003). Therefore, further evidence (anticipated vs. unanticipated) needs to be revealed and documented to comprehensively understand the landing posture control mechanism.

Predictably, in unexpected landing tasks, which may be partly due to poor technique, fatigue or unanticipated events, landing control may not be pre-planned adequately, placing the lower extremity in danger. To our knowledge, limited data are available regarding unexpected landing biomechanics. For instance, without vision, landings showed a $10 \%$ increase in ground reaction force and a $10 \%$ decrease in knee joint flexion compared to those under visual conditions (Santello et al., 2001). In an unexpected landing, the soft-tissue vibrations increase when a muscle reaction has less occurred, and the input frequency of the impact force is closer to the resonance frequency of the relevant soft-tissue compartments (Boyer and Nigg, 2006). Numerous studies have shown changed characteristics of soft tissue packages in response to muscle pre- and post-activation of the lower extremities (Boyer and Nigg, 2004, 2006; Nigg and Wakeling, 2001). A representative study indicated that the lower body adapted to different impact or input signals, mainly through muscle tuning, to minimize soft tissue vibrations during self-initiated ground contact (Boyer and Nigg, 2004). It has been found that when the available time to react is shorter than an individual's reaction time, the human movement strategy cannot be prepared sufficiently before landing (Stephenson et al., 2018). However, understanding how the soft tissue vibration changes in response to the impact force when a muscle reaction has not or less occurred is essential. Unfortunately, this mechanism has not been tested in an unexpected landing situation.

Therefore, this study aimed to determine the biomechanical changes in impact forces, lower extremity mechanics, soft tissue vibrations, and muscle activation during bipedal landings from self-initiated and unanticipated drops. We hypothesized that (1) the lower extremity would endure greater impact forces in an unanticipated drop landing (UDL) with an insufficient adjustment of landing posture shortly before and after ground contact, and (2) a greater soft tissue vibration would occur in response to less prepared muscle activation in unanticipated landings.

\section{Methods}

\section{Participants}

Twelve male collegiate basketball players (age: $23.7 \pm 2.7$ years; body height: $178.3 \pm 2.5 \mathrm{~cm}$; body mass: $70.1 \pm 4.6 \mathrm{~kg}$ ) with no musculoskeletal injuries in the lower extremities during the past 6 months participated in the study. A two-tailed ttest was executed via $G^{*}$ Power 3.1 software to determine whether a sample size of 12 participants was sufficient to minimize the probability of type II error for all the variables ( $\mathrm{P}=$ $80 \%$ at $\alpha=0.05$; ES $=0.4$ ) (Faul et al., 2007). Each participant signed an informed consent form prior to experimental testing.

Landing protocol

Before the formal tests, participants were required to complete a warm-up protocol consisting of 5 minutes of treadmill running at a self-selected speed followed by a set of static stretching exercises. Landing tasks included landing from a height of $30 \mathrm{~cm}$ in an anticipated drop jump (ADJ) and UDL (Figure 1). For ADJ, participants were asked to drop down on the force plates with the two feet separated, and then immediately jump as high as possible with the shortest contact time. For UDL, participants were instructed to stand on the landing platform, and the base of the platform was dropped manually by pulling a metal bolt from its slot to initiate the sudden drop landing movement. The time of removing the metal bolt was random to prevent the participants from guessing. During both 
landing movements, the participants were required to complete tasks with hands on the hips to reduce the influence of swinging. The participant was usually given 3-5 practice trials to become familiar with two landing conditions. Three successful trials for each condition were used for further analysis (Fu et al., 2013). Meanwhile, one experimenter was standing by the side of the participant to prevent him from getting injured or falling down.

In addition, uniform shoes (Shanghong Shoes Co. Ltd. ClassyVast. China) were provided to eliminate the cushioning influence from the shoes. They were equipped with a rubber outsole and a thin foam insole, but no midsole. Meanwhile, all the participants wore regular shorts and T-shirts with no tight fitting.

\section{Data collection}

Ground reaction forces

Vertical ground reaction forces were captured by two $90 \times 60 \mathrm{~cm}$ force plates $(9287 \mathrm{~B}$, Kistler Corporation, Switzerland) at a sampling rate of $1200 \mathrm{~Hz}$. The ground reaction force (GRF) and 3D kinematic data were sampled simultaneously using the Vicon system.

\section{Kinematics}

Sagittal kinematic data of the dominant lower extremity, which was the preferred leg for kicking a ball (Yeow et al., 2011b), were collected via a 3D motion capture system (MX13, Oxford Metrics, UK) at a sampling rate of $120 \mathrm{~Hz}$. Twenty-eight reflective markers with a diameter of $14.0 \mathrm{~mm}$ comprising the plug-in gait marker set were attached to the pelvis and the lower limb to define the hip, knee, and ankle joints (Fu et al., 2013).

\section{Accelerometry}

Two biaxial accelerometers (Biovision Corp., Wehrheim, Germany; $\pm 20 \mathrm{~g}$ ) were placed on the rectus femoris (RF) and biceps femoris (BF), and the corresponding vibrations of the quadriceps femoris (Quad) and hamstrings (Hams) were collected via the data acquisition system and DASYLab software (8.0, DATALOG GmbH, Mönchengladbach, Germany). One accelerometer (Biovision Corp., Wehrheim, Germany), with \pm 50 g measurement range, was attached to the heel cup of the shoe to determine the input signal (Boyer and Nigg, 2004). The accelerometers were placed onto the skin of the Quad and Hams using glue and secured with adhesive tape. The accelerometers were aligned with the first axis to be tangential to the skin and parallel to the longitudinal axis of the muscle, and the second axis was aligned normally to the skin (Boyer and Nigg, 2006). Detailed description of placement was presented previously by Fu et al. (2013).

Surface electromyography (EMG)

A Biovision EMG system (Biovision, Wehrheim, Germany) was used to record the EMG signals from the tibialis anterior (TA), lateral gastrocnemius (LG), $\mathrm{RF}$, and $\mathrm{BF}$ of the dominant leg. Surface electrodes $(\mathrm{Ag} / \mathrm{AgCl})$ were placed on the muscle bellies after the skin was carefully prepared (shaved and cleaned with alcohol) to reduce skin impedance. The EMG and acceleration signals were recorded simultaneously at a sampling rate of $1200 \mathrm{~Hz}$ using the data acquisition system and DASYLab software (8.0, DATALOG GmbH, Moenchengladbach, Germany). The data acquisition system and the Vicon system were synchronized by an external trigger generated by a customized signal generator.

\section{Data analysis}

Impact forces

The impact phase was defined as the time interval from the initial foot contact to the maximum of the vertical GRF. The vertical GRF was normalized by body weight (BW). The characteristics of the input signal during the impact phase included the peak vertical GRF ( $\left.\mathrm{F}_{\text {zmax }}\right)$, the occurrence time of $\mathrm{F}_{\mathrm{zmax}}\left(\mathrm{t}_{\mathrm{F}}\right)$, the peak loading rate $\left(G_{z}\right)$, the occurrence time of $G_{z}\left(t_{G}\right)$, and GRF frequency (fGRF). The $f_{G R F}$ was determined by Equations (A. 1) and (A. 2) (Fu et al., 2013):

$$
\begin{gathered}
\mathrm{G}_{\mathrm{z}, \mathrm{ave}}=\lim _{\Delta \mathrm{t} \rightarrow 0} \frac{\Delta \mathrm{F}}{\Delta \mathrm{t}} \\
\mathrm{f}_{\mathrm{GRF}}=\frac{1}{2\left(\mathrm{~F}_{\mathrm{zmax}} / \mathrm{G}_{\mathrm{z}, \text { ave }}\right)}
\end{gathered}
$$

where $F_{z \max }$ is the peak value of the vertical GRF, $\mathrm{G}_{\mathrm{z}, \text { ave }}$ is the average loading rate between 20 to $80 \%$ of the impact phase.

\section{Kinematics}

The sagittal plane kinematic data were filtered at $7 \mathrm{~Hz}$ using a fourth-order, zero-lag lower-pass Butterworth filter (Fu et al., 2013). The joint angles of the hip, knee, and ankle $\left(\theta_{0}\right)$ of the touchdown (the definition of the joint angle was shown in Figure 2 and change in the joint angle $(\Delta \theta)$ in degrees from the touchdown to maximum 
knee flexion for the three joints was determined via Visual 3D software). The maximum vertical displacement of the center of mass $(\Delta y)$ and vertical stiffness $\left(k_{\text {vert }}\right)$ were used to describe changes in kinematics. The vertical stiffness ( $\left.k_{\text {vert }}\right)$ is often used to describe linear movements that occur in the vertical direction such as hopping and jumping (considering the lower extremity as a simple mass-spring model) (Butler et al., 2003; Jordan et al., 2018). kvert was calculated with the following Equation (B) (Mcmahon and Cheng, 1990):

$$
k_{\text {vert }}=\frac{F_{z \max }}{\Delta y}
$$

where $\mathrm{F}_{\mathrm{zmax}}=$ maximal vertical impact force, $\Delta y=$ change in vertical displacement of the center of mass.

Soft tissue vibrations

The main variables included peak soft tissue acceleration $\left(a_{\text {peak }}\right)$, occurrence time of apeak $\left(\mathrm{t}_{\mathrm{a}}\right)$, domain frequency $\left(f_{\mathrm{v}}\right)$, and damp coefficient $(c)$ in ADJ and UDL (Wakeling and Nigg, 2001). The vibration frequency and damping coefficients are natural vibration characteristics of soft tissues packages that depend on muscle activations, muscle length, shortening velocity, etc. (Nigg and Wakeling, 2001; Wakeling and Nigg, 2001). The vibration damping characteristics were determined by the following model $(\mathrm{C})$ :

$$
\mathrm{s}=a e^{-c t} \sin \left(2 \pi f_{v} t+\varphi\right)
$$

where $s$ is the measured acceleration signal, a is the amplitude of acceleration, $c$ is the damping coefficient, $f_{\mathrm{v}}$ is the domain vibration frequency of soft tissue (damped), and $\varphi$ is the phase coefficient.

Muscle activation

The EMG data were analyzed using DASYLab software (8.0, DATALOG $\mathrm{GmbH}$, Mönchengladbach, Germany), and band-pass filtered from 10 to $400 \mathrm{~Hz}$ using a fourth order, zero-lag Butterworth filter. The EMG amplitudes (root mean square, EMGrms) were normalized as a percentage of the highest value recorded during the entire contact phase of all ADJ trials (Ruan and $\mathrm{Li}, 2010)$ using the following Equation (D):

$$
\mathrm{EMG}_{R M S}=\sqrt{\frac{1}{T} \int_{t}^{t+T} E M G^{2}(t) d t}
$$

where $t$ is the onset of the signal and $T$ is the time interval of each phase. In this study, the phases were defined as pre-activation (- 50 to $0 \mathrm{~ms}$ before the touchdown), post-activation (0 to $50 \mathrm{~ms}$ after the touchdown), and downward (touchdown to the occurrence of maximum knee flexion).

\section{Statistics}

Paired $t$-tests were used to examine the effects of landing tasks on landing kinematics, impact forces, soft tissue vibrations, and muscle activation (13.0, SPSS Inc., Chicago, IL, U.S.A.). The significance level $\alpha$ was set at 0.05 .

\section{Results}

Impact forces

UDL showed significantly larger $F_{z m a x}$, shorter $t_{F}$, and also larger $\mathrm{G}_{\mathrm{z}}$ than ADJ. $f_{\mathrm{GRF}}$ also significantly increased in UDL (Figure 3).

Joint angle and vertical stiffness

Compared with UDL, the $\theta_{0}$ of the knee and hip joints significantly decreased in ADJ. During the downward phase, ADJ exhibited significantly increased $\Delta L(100 \%)$ and decreased $\mathrm{k}_{\text {vert }}(70.2 \%)$ (Table 1).

\section{Soft tissue vibrations}

The Quad and Hams had significantly lower $a_{\text {peak }}$ in $\mathrm{ADJ}$ compared to UDL, whereas $c$ was significantly greater in ADJ than in UDL. However, no significant differences were found in $t_{a}$ of Quad and Hams, and $f_{\mathrm{v}}$ between the two landing conditions (Table 2).

EMG

During pre-activation, EMGRMs of LG, RF, and $\mathrm{BF}$ were significantly greater in ADJ than in UDL. During post-activation and downward phases, ADJ also showed significant increases in activation levels in all muscle groups $(\mathrm{p}<.05$, Figure 4).

\section{Discussion}

The present study investigated the effects of movement control on impact forces, kinematics, soft tissue vibrations, and EMG between ADJ and UDL. The $F_{z \max }, G_{z}$, and $f_{\text {GRF }}$ were all lower in ADJ than in UDL. ADJ also showed more joint flexion, increased $c$ of soft-tissue vibrations, and a higher level of muscles activation than UDL. These findings supported our hypothesis that the lower extremity would demonstrate greater shock magnitude of impact forces with less muscle 
activation before and after foot ground contacting in UDL which also caused greater soft tissue vibrations.

Impact forces

In $\mathrm{ADJ}$, more time was required to obtain significantly lower peak vertical GRF, which caused a decrease in the loading rate.

Falling from a distance above the floor, the magnitude of the collision and the amount of negative muscular work performed depended on the landing strategy (Gambelli et al., 2016). The self-selected landing strategy lies in between stiff and soft landing, which may reflect how the participants value the trade-offs between economy and discomfort or pain caused by a large impact force
(Zelik and Kuo, 2012). If magnitude of the ground reaction force encountered during a landing is too great, and the musculoskeletal system is unable to disperse the forces effectively, then the probability of injury occurrences increases dramatically (Standing and Maulder, 2015), frequently leading to acute injuries (Beynnon et al., 2005) and overuse damage (Borowski et al., 2008; Dick et al., 2007) caused by landing.
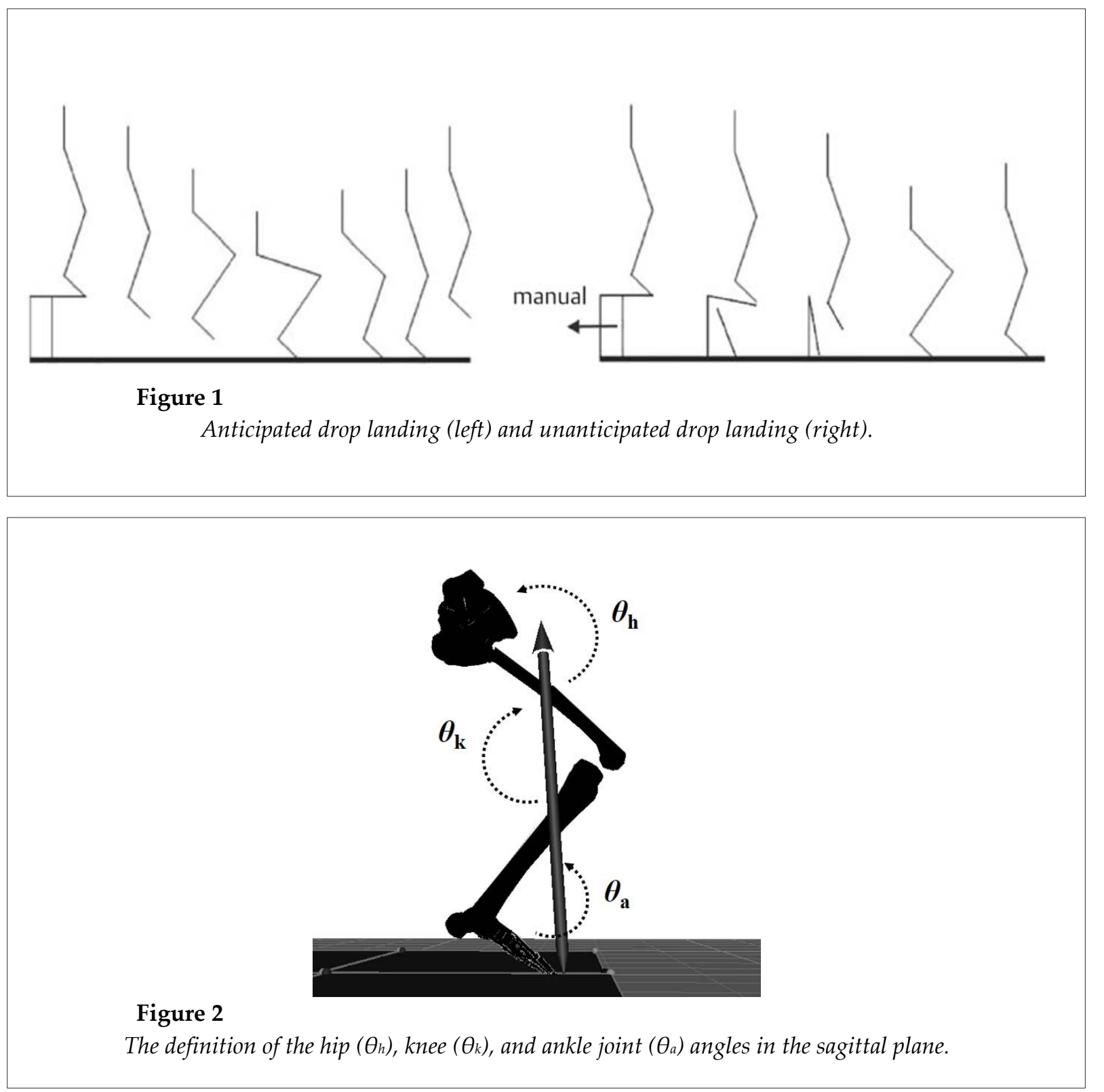

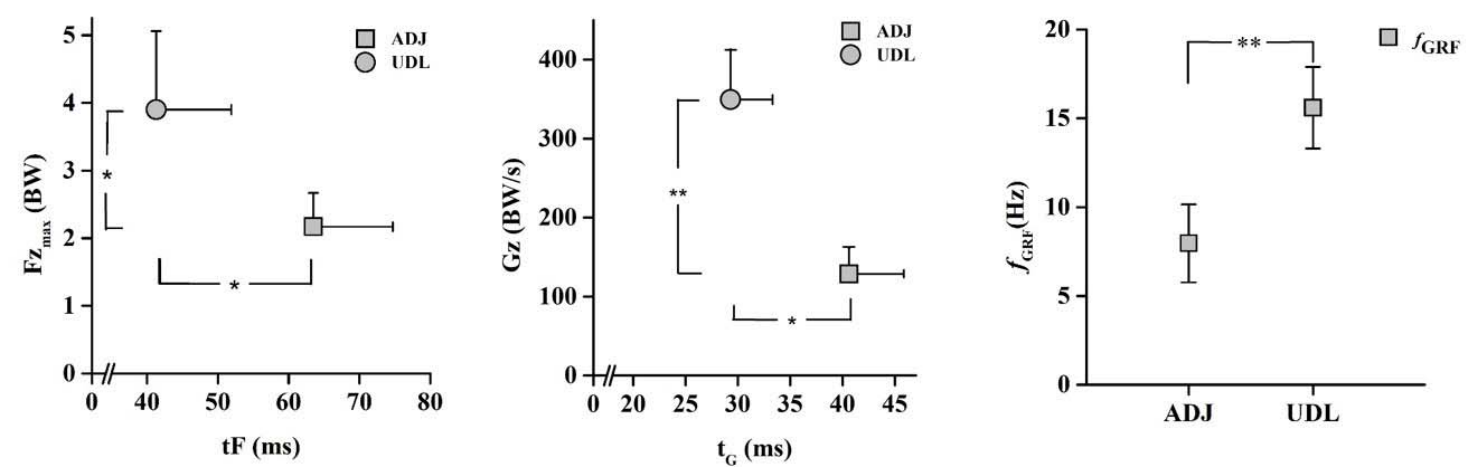

Figure 3

The peak vertical $G R F\left(F_{z \max }\right)$ \& occurrence time of $F_{z \max }\left(t_{F}\right)$, the peak loading rate $\left(G_{z}\right)$ $\mathcal{E}$ occurrence time of $G_{z}\left(t_{G}\right)$, and $G R F$ frequency $\left(f_{G R F}\right)$ in $A D J \mathcal{E} U D L$.

* Significant difference between ADJ and UDL with $p<0.05$.

** Significant difference between ADJ and UDL with $p<0.01$.

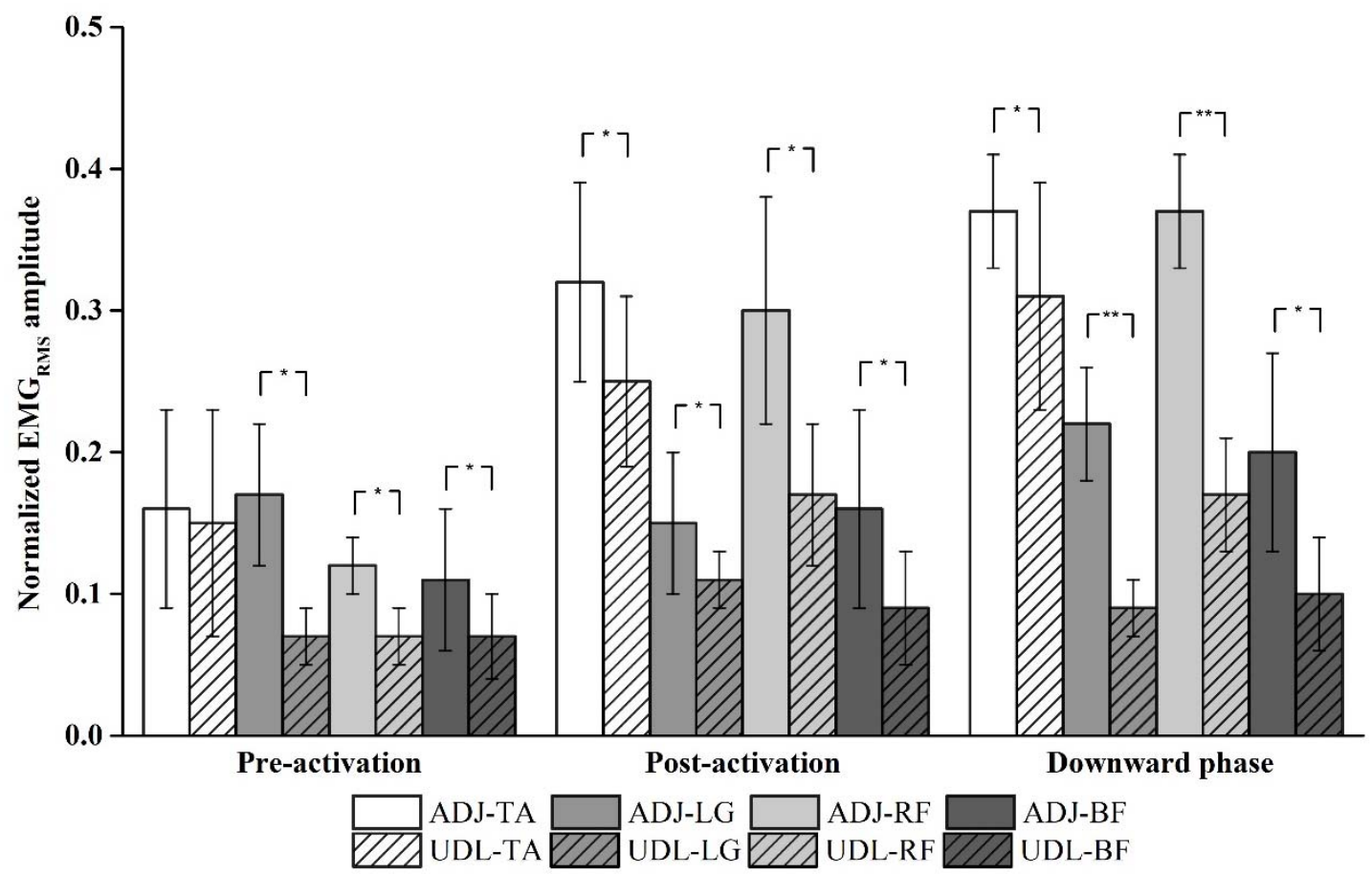

Figure 4

Normalized EMGRMs amplitude of tibialis anterior (TA), lateral gastrocnemius (LG), rectus femoris $(R F)$ and biceps femoris $(B F)$ of pre-activation, post-activation and the downward phase in ADJ \& UDL.

* Significant difference between ADJ and UDL with $p<0.05$.

** Significant difference between ADJ and UDL with $p<0.01$. 
Table 1

Joint kinematics in $A D J \mathcal{E} U D L$.

\begin{tabular}{|c|c|c|c|}
\hline Variables & Joint & ADJ & UDL \\
\hline \multirow{3}{*}{$\theta_{0}(\stackrel{\mathrm{o}}{\mathrm{N}})$} & Hip & $136.2 \pm 11.9^{*}$ & $156.9 \pm 12.7$ \\
\hline & Knee & $155.3 \pm 6.8^{*}$ & $164.9 \pm 3.8$ \\
\hline & Ankle & $137.9 \pm 5.0$ & $136.0 \pm 6.0$ \\
\hline \multirow{3}{*}{$\Delta \theta\left({ }^{\circ}\right)$} & Hip & $29.9 \pm 13.1^{*}$ & $10.4 \pm 4.0$ \\
\hline & Knee & $55.3 \pm 12.1^{*}$ & $35.2 \pm 5.8$ \\
\hline & Ankle & $41.3 \pm 6.4^{*}$ & $32.2 \pm 5.2$ \\
\hline \multicolumn{2}{|c|}{$\Delta L(\mathrm{~m})$} & $0.26 \pm 0.05^{*}$ & $0.13 \pm 0.02$ \\
\hline \multicolumn{2}{|c|}{$k_{\text {vert }}(\mathrm{BW} / \mathrm{m})$} & $9.0 \pm 3.7^{* *}$ & $30.2 \pm 5.0$ \\
\hline
\end{tabular}

$\theta_{0}$, joint angles of touchdown; $\Delta \theta$, change in joint angle in degrees;

$\Delta L$, the vertical changes in maximum length; $k_{\text {vert, }}$ vertical stiffness.

* Significant difference between ADJ and UDL with $p<0.05$.

** Significant difference between ADJ and UDL with $p<0.01$.

\section{Table 2}

Characteristics of soft tissue vibration in $A D J \mathcal{E} U D L$.

\begin{tabular}{cccc}
\hline Variables & Muscle & ADJ & UDL \\
\hline \multirow{2}{*}{$a_{\text {peak }}(\mathrm{g})$} & Quad & $7.82 \pm 3.2^{* *}$ & $15.66 \pm 4.3$ \\
& Hams & $4.42 \pm 1.9^{*}$ & $7.41 \pm 3.2$ \\
\hline \multirow{2}{*}{$\mathrm{t}_{a}(\mathrm{~ms})$} & Quad & $62.3 \pm 28.7$ & $53.9 \pm 20.1$ \\
& Hams & $56.6 \pm 26.6$ & $49.1 \pm 23.1$ \\
\hline \multirow{2}{*}{$f_{v}(\mathrm{~Hz})$} & Quad & $13.83 \pm 1.6$ & $14.24 \pm 2.0$ \\
& Hams & $16.17 \pm 3.1$ & $19.75 \pm 3.2$ \\
\hline \multirow{2}{*}{$c\left(\mathrm{~s}^{-1}\right)$} & Quad & $13.86 \pm 3.5^{*}$ & $10.67 \pm 1.3$ \\
& Hams & $11.68 \pm 5.7^{*}$ & $9.37 \pm 4.2$ \\
\hline
\end{tabular}

$a_{\text {peak, }}$ peak soft-tissue acceleration; $t_{a}$, the occurrence time of $a_{\text {peak }}$; $f_{v}$, the domain frequency;

c, damp coefficient; quad, quadriceps femoris; hams, hamstrings.

* Significant difference between ADJ and UDL with $p<0.05$.

** Significant difference between ADJ and UDL with $p<0.01$. 
The characteristics of the impact force are influenced by drop heights, kinematics of lower extremity, and the landing surface. In the present study, the landing strategy was the main factor affecting the ground reaction force without considering the test environments. Thus, stiff landings caused a greater ground reaction force than soft landings (Kim and Jeon, 2016). Moreover, soft and stiff landings had relatively large and small amounts of knee flexion, respectively, during the floor contact phase. Our results demonstrated that the lower vertical impact force with higher hip and knee flexion angles in ADJ suggested that soft landing reduced the impact stress on the body tissues with hip and knee muscles absorbing more energy compared with the stiff landing (Devita and Skelly, 1992). Furthermore, EMG activity, which is related to the landing from a step or a jump to the ground, began before the movement of the landing. This observation reflects a strategy to prepare the muscles-tendon complex for a rapid, forceful stretch occurring after foot contact and the subsequent joint flexion (Jones and Watt, 1971). Given that the amplitude and frequency of impact force could be altered by changes in leg kinematics and joint stiffness at ground contact (Wakeling and Nigg, 2001), landing from an unexpected fall may cause any reflex muscular activity to occur too late to decelerate the body mass (Jones and Watt, 1972). The present results confirmed that UDL induced a stiff posture, which increased the risk of injury.

\section{Kinematics}

In this study, hip and knee joints showed significantly lower joint angles at contact, more changes in vertical leg length, and less leg stiffness in ADJ compared with UDL.

In a landing task, the shock experienced by the body during ground contact must be attenuated and dispersed mostly by the lower extremities structure or kinematics (Tamura et al., 2016). In humans, the effective movement reflection of external impact force depends on motor learning at early growth processes, with proficiency at $10-12$ years of age, experiences with an activity, and task familiarity (Magalhaes and Goroso, 2009). Different magnitude of impacts needs varying amplitudes of muscle force to control joint flexion.

Muscle pre-activation during the flight phase of landing was used to indicate a continuous build-up of muscle force occurring before the touchdown to (1) form initial stiffness of the contractile component, which enables the use of elastic energy from muscle-tendon structures in conjunction with the muscle contractile property, and (2) provide adequate deceleration of joint flexion during dynamic activities, which may be a mechanism acting to protect the musculoskeletal system from injury (Vladimir et al., 2008). As expected, our findings showed that the significantly decreased pre-activation EMG in UDL led to a stiff landing, with the hip and knee joints showing significantly lower flexion at the touchdown phase, lower changes in the joint angle, and relatively higher leg stiffness, which caused greater impact of ground reaction force compared with ADJ (Kim and Jeon, 2016).

Therefore, landings from an unexpected fall would make any reflex muscular activity resulting from the landing event occur too late to be of use in decelerating the body mass (Jones and Watt, 1971). Low post-activation EMG indicated that the body controlled joint flexion during the downward phase less actively to cushion the impact compress on the body and influenced the ability of the lower extremities to contribute to energy dissipation (Zhang et al., 2000).

Soft tissue vibration and muscle activation

Our results showed that ADJ had significantly decreased $a_{\text {peak }}$ and increased $c$ in Quad and Ham compared with UDL without any differences in ta and $f_{v}$. ADJ showed certain effects on attenuating the soft tissue vibration of the quadriceps femoris and hamstrings.

As a result of the rapid deceleration of the leg during landing, vibrations of the soft tissue compartment muscles, fascia, surrounding tissue and skin of the leg were initiated. The initial magnitude of these vibrations was large, but they were heavily damped (Boyer and Nigg, 2004). Wakeling and Nigg (2001) revealed that characteristics of natural frequencies and damping properties of soft tissues vibration depended on muscle activity, which is called muscle tuning. In this process, soft tissue vibrations are minimized by shifting the free vibration frequencies away from those of the impact forces (Wakeling and Nigg, 2001). The frequency and damping coefficients of vibrations in soft tissues of the lower extremities increased 
by increasing in muscle force production and muscle shortening velocity (Wakeling and Nigg, 2001).

Muscle activation occurs during the flight phase in preparation for the impact before the touchdown. The build-up of muscle force in the pre-contact phase forms initial stiffness of the contractile component from the muscle-tendon structure and provides sufficient deceleration of joint flexion after the touchdown (Vladimir et al., 2008). Our EMG results suggested that inappropriate levels of muscle stiffness occurred more frequently in unanticipated falling, in which time was insufficient to build-up "preparatory" muscle activity before the touchdown (Santello, 2005). The Quad and Hams had lower damping coefficients, so the maximal amplitude of vibration was greater in UDL than in ADJ. However, the domain frequencies of Quad and Hams were not significant between ADJ and UDL, which was in contrast to findings of previous studies. The muscles possibly attenuated the amplitude of vibration by increasing the damping coefficient without natural frequency changes. The internal mechanisms underlying how the soft tissue damping coefficient affects the amplitude of vibration and domain frequency during landing need to be further explored.

The main limitation of this study was the inclusion of only male athletes and the use of only one height. Although the current short time window was a design to elicit unanticipated landings, future work is still needed to determine whether professional athletes respond differently under unexpected landing condition with different heights (e.g., $40 \mathrm{~cm}, 50 \mathrm{~cm}$, and $60 \mathrm{~cm}$ ), and to understand the gender effects.

\section{Conclusion}

Humans can modulate landing strategies by increasing joint flexion, decreasing $k_{\mathrm{vert}}, \mathrm{F}_{\mathrm{zmax}}$, and $\mathrm{G}_{z}$, as well as attenuating $a_{\text {peak }}$ with greater $c$, and enhancing muscle activation to achieve a safe and smooth landing, as in ADJ. By contrast, when one is unable to make a timely prediction, like in UDL, low muscle activation occurs such that the musculoskeletal system endures increased impact forces and soft tissue vibrations, which may lead to landing-related injuries.

\section{Acknowledgements}

This work was supported by the National Natural Science Foundation of China (11772201, 81572213); National Key Research and Development Program of China (2018YFF0300500); Shanghai Committee of Science and Technology (17080503300); the Talent Development Fund of Shanghai Municipal (2018107).

\section{References}

Almonroeder TG, Garcia E, Kurt M. The effects of anticipation on the mechanics of the knee during singleleg cutting tasks: A systematic review. Int J Sports Phys Ther, 2015; 10: 918

Beynnon BD, Vacek PM, Murphy D, Alosa D, Paller D. First-time inversion ankle ligament trauma: the effects of sex, level of competition, and sport on the incidence of injury. Am J Sports Med, 2005; 33: 1485-1491

Borowski LA, Yard EE, Fields SK, Comstock RD. The epidemiology of US high school basketball injuries, 2005-2007. Am J Sports Med, 2008; 36: 2328-2335

Boyer KA, Nigg BM. Muscle activity in the leg is tuned in response to impact force characteristics. J Biomech, 2004; 37: 1583-1588

Boyer KA, Nigg BM. Muscle tuning during running: implications of an un-tuned landing. J Biomech Eng, 2006; $128: 815-822$

Brüggemann G-P, Brüggemann L, Heinrich K, Müller M, Niehoff A. Biological tissue response to impact like mechanical loading. Footwear Sci, 2011; 3: 13-22 
Butler RJ, Iii HPC, Davis MC. Lower extremity stiffness: implications for performance and injury. Clin Biomech, 2003; 18: 511-517

Decker MJ, Torry MR, Wyland DJ, Sterett WI, Steadman JR. Gender differences in lower extremity kinematics, kinetics and energy absorption during landing. Clin Biomech, 2003; 18: 662-669

Del Coso J, Herrero H, Salinero JJ. Injuries in Spanish female soccer players. J Sport Health Sci, 2018; 7: 183190

Delahunt E, Monaghan K, Caulfield B. Changes in lower limb kinematics, kinetics, and muscle activity in subjects with functional instability of the ankle joint during a single leg drop jump. J Orthop Res, 2006; 24: 1991-2000

Devita P, Skelly WA. Effect of landing stiffness on joint kinetics and energetics in the lower extremity. Med Sci Sports Exerc, 1992; 24: 108-115

Dick R, Hertel J, Agel J, Grossman J, Marshall SW. Descriptive epidemiology of collegiate men's basketball injuries: National Collegiate Athletic Association Injury Surveillance System, 1988-1989 through 20032004. J Athl Train, 2007; 42: 194

Dixon SJ, Collop AC, Batt ME. Surface effects on ground reaction forces and lower extremity kinematics in running. Med Sci Sport Exer, 2000; 32: 1919-1926

Faul F, Erdfelder E, Lang AG, Buchner A. G*Power 3: A flexible statistical power analysis program for the social, behavioral, and biomedical sciences. Behav Res Methods, 2007; 39: 175-191

$\mathrm{Fu} \mathrm{W,} \mathrm{Liu} \mathrm{Y,} \mathrm{Zhang} \mathrm{S.} \mathrm{Effects} \mathrm{of} \mathrm{footwear} \mathrm{on} \mathrm{impact} \mathrm{forces} \mathrm{and} \mathrm{soft} \mathrm{tissue} \mathrm{vibrations} \mathrm{during} \mathrm{drop} \mathrm{jumps} \mathrm{and}$ unanticipated drop landings. Int J Sports Med, 2013; 34: 477-483

Gambelli CN, Theisen D, Willems PA, Schepens B. Human motor control of landing from a drop in simulated microgravity. J Appl Physiol, 2016; 121: jap.00305.02016

Golas A, Wilk M, Statsny P, Maszczyk A, Pajerska K, Zajac A. Optimizing Half Squat Post Activation Potential Load In Squat Jump Training For Eliciting Relative Maximal Power In Ski Jumpers. J Strength Cond Res, 2017; doi: 10.1519/JSC.0000000000001917 [Epub ahead of print]

Jones GM, Watt DGD. Observations on the control of stepping and hopping movements in man. J Physiol, 1971; 219: 709-727

Jones GM, Watt DGD. Muscular control of landing from unexpected falls in man. J Physiol, 1972; 219: 729-737

Jordan MJ, Aagaard P, Herzog W. A comparison of lower limb stiffness and mechanical muscle function in ACL-reconstructed, elite, and adolescent alpine ski racers/ski cross athletes. J Sport Health Sci, 2018; 7: 416-424

Kim K, Jeon K. Comparisons of knee and ankle joint angles and ground reaction force according to functional differences during single-leg drop landing. J Phys Ther Sci, 2016; 28: 1150-1154

Magalhaes FH, Goroso DG. Preparatory EMG activity reveals a rapid adaptation pattern in humans performing landing movements in blindfolded condition. Percept Mot Skills, 2009; 109: 500-516

Mandelbaum BR, Silvers HJ, Watanabe DS, Knarr JF, Thomas SD, Griffin LY, Kirkendall DT, Garrett JW. Effectiveness of a neuromuscular and proprioceptive training program in preventing anterior cruciate ligament injuries in female athletes: 2-year follow-up. Am J Sports Med, 2005; 33: 1003-1010

Mcmahon TA, Cheng GC. The mechanics of running: how does stiffness couple with speed? J Biomech, 1990; 23: $65-78$

Moran KA, Wallace ES. Eccentric loading and range of knee joint motion effects on performance enhancement in vertical jumping. Hum Mov Sci, 2007; 26: 824-840

Nigg BM, Wakeling JM. Impact forces and muscle tuning: a new paradigm. Exerc Sport Sci Rev, 2001; 29: 3741

Ruan M, Li L. Approach run increases preactivation and eccentric phases muscle activity during drop jumps from different drop heights. J Electromyogr Kinesiol, 2010; 20: 932-938

Santello M. Review of motor control mechanisms underlying impact absorption from falls. Gait Posture, 2005; 21: 85-94

Santello M, Mcdonagh MJ, Challis JH. Visual and non-visual control of landing movements in humans. J Physiol, 2001; 537: 313-327

Self BP, Paine D. Ankle biomechanics during four landing techniques. Med Sci Sports Exerc, 2001; 33: 1338 
Shultz SJ, Schmitz RJ, Tritsch AJ, Montgomery MM. Methodological considerations of task and shoe wear on joint energetics during landing. J Electromyogr Kines, 2011; 22: 124-130

Standing RJ, Maulder PS. A Comparison of the Habitual Landing Strategies from Differing Drop Heights of Parkour Practitioners (Traceurs) and Recreationally Trained Individuals. J Sports Sci Med, 2015; 14: 723

Stephenson ML, Hinshaw TJ, Wadley HA, Zhu Q, Wilson MA, Byra M, Dai B. Effects of timing of signal indicating jump directions on knee biomechanics in jump-landing-jump tasks. Sports Biomech, 2018; 17: 67-82

Tamura A, Akasaka K, Otsudo T, Sawada Y, Okubo Y, Shiozawa J, Toda Y, Yamada K. Fatigue Alters Landing Shock Attenuation During a Single-Leg Vertical Drop Jump. Orthop J Sports Med, 2016; 4: 2325967115626412

Teh J, Firth M, Sharma A, Wilson A, Reznek R, Chan O. Jumpers and fallers: a comparison of the distribution of skeletal injury. Clin Radiol, 2003; 58: 482-486

Vladimir M, Ilic DB, Nenad J, Zeljko R, Djordje S. Pre-activity modulation of lower extremity muscles within different types and heights of deep jump. J Sports Sci Med, 2008; 7: 269-278

Wakeling JM, Nigg BM. Modification of soft tissue vibrations in the leg by muscular activity. J Appl Physiol, 2001; 90: 412-420

Yeow $\mathrm{CH}$, Lee PV, Goh JC. An investigation of lower extremity energy dissipation strategies during singleleg and double-leg landing based on sagittal and frontal plane biomechanics. Hum Mov Sci, 2011a; 30: 624-635

Yeow $\mathrm{CH}$, Lee PVS, Goh JCH. Shod landing provides enhanced energy dissipation at the knee joint relative to barefoot landing from different heights. Knee, 2011b; 18: 407-411

Zelik KE, Kuo AD. Mechanical Work as an Indirect Measure of Subjective Costs Influencing Human Movement. Plos One, 2012; 7: e31143

Zhang S, Bates BT, Dufek JS. Contributions of lower extremity joints to energy dissipation during landings. Med Sci Sports Exerc, 2000; 32: 812-819

\section{Corresponding authors:}

\section{Weijie Fu, PhD.}

Key Laboratory of Exercise and Health Sciences of the Ministry of Education

Shanghai University of Sport

200438 Shanghai, China

Tel.: +86-21-51253239; Fax: +86-21-51253380

Email: fuweijie@sus.edu.cn

\section{Yu Liu, PhD}

Key Laboratory of Exercise and Health Sciences of the Ministry of Education Shanghai University of Sport

200438 Shanghai, China

Tel.: +86-21-51253571; Fax: +86-21-51253380

Email: yuliu@sus.edu.cn 